\title{
Food Quality and Preference
}

\section{What can cognitive psychology and sensory evaluation learn from each other?}

\author{
Hervé Abdi \\ The University of Texas at Dallas, PO Box 830688, Richardson, TX 75083 0688, USA
}

\begin{abstract}
Two questions are addressed in this paper: What can cognitive psychology bring to sensory evaluation? And what can cognitive psychology learn from sensory evaluation? In the first part, I will argue that one important contribution from psychology to sensory evaluation is to interpret flavor as a cognitively unified system made of three anatomically separated systems (smell, taste, and the trigeminal system). In the second part, I will argue that the applied field of sensory evaluation stresses the importance of using ecologically valid, naturalistic stimuli. Sensory evaluation also provides results that challenge accepted interpretations in psychology, especially in the field of evaluation of expertise.
\end{abstract}

(C) 2002 Elsevier Science Ltd. All rights reserved.

Keywords: Cognition; Cognitive psychology; Expertise; Face processing; Flavor; Halo-dumping; Odor; Odor/taste interaction; Olfaction; Orbitofrontal cortex; Sensory evaluation; Sensory integration; Smell; Synesthesia; Taste; Trigeminal system

\section{Introduction}

Cognitive psychology, or more broadly cognitive science, aims to understand how people or machines acquire, store, retrieve, and use knowledge and information. Sensory evaluation analyzes how food products are perceived (see, e.g., Lawless \& Heymann, 1999). Given their definitions, it would seem that these two disciplines should interact closely. But, in fact, they have essentially existed without much interaction. Following early attempts aimed at integrating these fields (see, e.g., Koster, 1983) this unfortunate state of affairs has recently started to change.

Rather than reviewing all these exchanges, I will illustrate (admittedly, in an idiosyncratic way) that they show that cognitive psychology can provide some theoretical frameworks for sensory evaluation; and that, in turn, sensory evaluation can provide content for some psychological questions. This paper is structured accordingly: A first part exposes the contributions of cognitive psychology to sensory evaluation, and a second part exposes the contribution of sensory evaluation to cognitive psychology

What can sensory evaluation learn from cognitive psychology? I will start with an overview of the neuroanatomy of the structures involved in the perception of

E-mail address: herve@utdallas.edu (H. Abdi). chemical stimuli: taste, smell, and trigeminal systems. These are obviously separate systems with separate functions. Therefore it is tempting to infer that these systems are also cognitively independent. I will put this contention in parallel with vision. In vision, we find the same type of systemic dissociation between movement, position, and identification of objects. However, subjects do not experience these qualities as being independent properties of objects perceived in the world. Similarly, I will argue that the subjective experience of flavor (what naive subjects call taste) integrates information from smell, taste, and the trigeminal system. I will then illustrate the implications of this integration for sensory evaluation. I conclude this part by suggesting that the orbitofrontal cortex may be the brain structure that performs this integration.

What can cognitive psychology learn from sensory evaluation? Recently, cognitive psychology has started to recognize the importance of working with naturalistic stimuli. This trend has been particularly clear with nonverbal stimuli such as faces or music because, here, the use of artificial stimuli can lead to erroneous interpretations. Sensory evaluation shows that the scientific need for control of the stimulus should not lead to the destruction of its natural complexity and its ecological validity. In addition, sensory evaluation opens new fields of investigation for cognitive psychologists by drawing their attention to neglected but important senses. 


\section{Part I: what can sensory evaluation learn from cognitive psychology?}

\subsection{Olfactory system}

For smell, obviously, everything starts in the nose when an odorant substance binds to the membrane of the olfactory cells (see, e.g., Kandel, Schwartz, \& Jessell, 2000). These specialized neurons are located in a small patch of tissue in the upper part of the nasal cavity. They are bipolar cells whose dendrites - peppered with specialized receptors - are floating in a layer of mucous. The axons of these olfactory cells cross the cribiform ethmoid bone to make synapses with neurons called mitral cells in the olfactory bulb. These connections are made in small globular structures called glomeruli. Glomeruli are connected to each other by the periglomerular cells. These cells, in conjunction with the deeper granule cells, seem to play a róle similar to the róle of the horizontal cells in the retina (i.e., to use lateral inhibition in order to "sharpen the signal;" see, e.g., Laurent, Stopfer, Friedrich, Rabinovich, Volkovskii, \& Abarbanel, 2001). The axons of the mitral cells constitute the olfactory tract which connects the olfactory bulb to the brain. The axons of the olfactory tract will then reach directly several structures including the anterior olfactory nucleus, the olfactory tubercle, the pyriform cortex, and the entorhinal cortex. From these structures the olfactory information can reach the amygdala, the hippocampus, the hypothalamus, the thalamus, and the orbitofrontal cortex (Doty, 2001; see Figs. 1 and 2). The hypothalamus

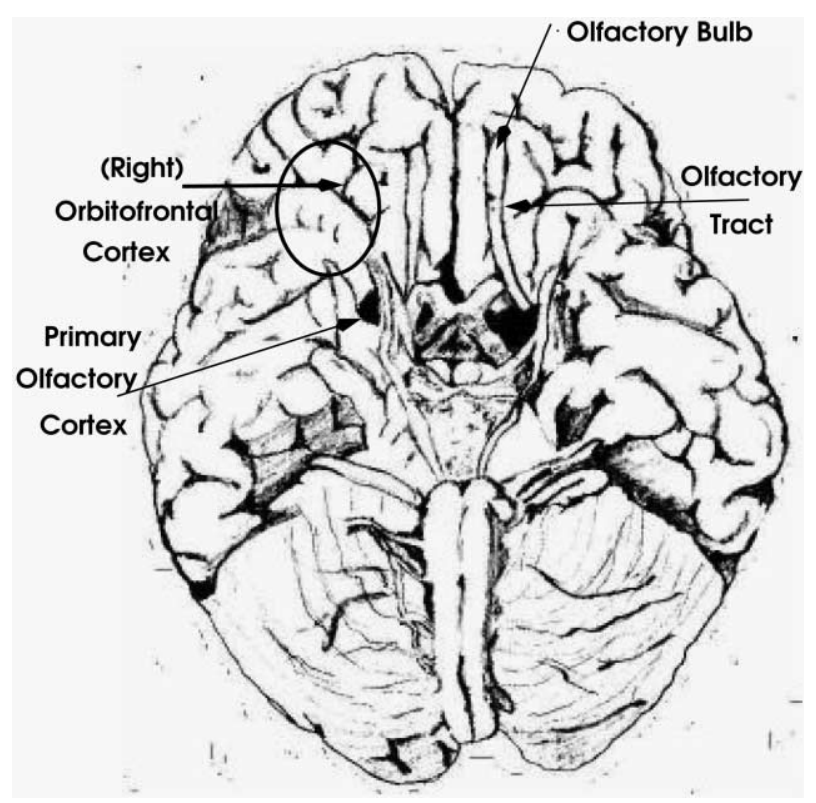

Fig. 1. A ventral view of the brain showing the location of the orbitofrontal cortex along with some other ventral brain structures important for flavor.

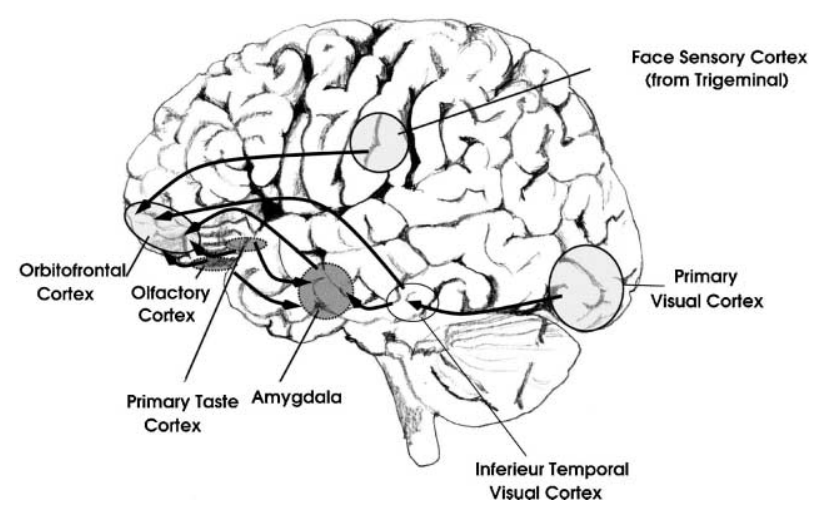

Fig. 2. Connections to the orbitofrontal cortex: the orbitofrontal cortex receives information from the sensory (smell, taste, trigeminal, touch, vision) and emotional centers (amygdala) of the brain involved in creating the cognitively unified sensation of flavor.

and the amygdala are involved in the processing of emotions; the hippocampus participates in storage and retrieval of memories, the thalamus and the orbitofrontal cortex are involved in perception and cognition.

How are odors coded at the level of the sensory cells? We currently believe that one olfactory cell expresses only one out of maybe one thousand odor receptors on the cilia which expand from its dendrites (Holley, 1999; Mombaerts, 2001). The receptors themselves are not narrowly specific, which means that one receptors binds several odorants and one odorant binds with several different receptors (some receptors maybe more specific than some others, which may explain some specific anosmias as they can occur when one narrowly tune receptor is deficient).

Therefore, a given odorant is coded by a pattern of activated cells (Kauer \& White, 2001). At the level of the olfactory bulb, one glomerulus collects information from olfactory cells expressing the same receptor. Here again (cf. the olfactory cells), an odor is coded by the pattern of activation of the population of glomeruli.

To understand a perceptual system we try, in general, to identify the physical properties that determine its response (e.g., the wavelength of light determines the perception of color). So the question for smell has been to find the physical or chemical properties that determine the odor of a molecule or a mixture. And essentially, that effort has failed for two main reasons. First, similar molecules can have very different odors (e.g., the two isomers alpha-ionone and beta-ionone smell respectively like violet and berries). Second, very different molecules can have the same odor (e.g., isoamyl acetate and hept-4-in-2-ol both smell like banana). So, odors are likely to be represented by patterns of activation of the cells. In fact, the subjective classification of odors seems to reflect more the ecological function of odors (e.g., a rotten smell means: "do not eat it") than chemical properties per se. 


\subsection{Gustatory system}

Taste buds, which contain taste cells, are localized in the tongue in structures called papillae. The fibers coming from the taste buds are collected in the facial nerve and the glossopharyngeal nerve. They make a first synapse in the nucleus tractus solitarus located in the brain stem. Then, they make a second synaptic contact at the level of the thalamus and finally reach the gustatory cortex (see, Shepherd, 1994).

The way taste is coded is still a matter of some controversy, as two theories compete. The first one, called population coding theory (or "across fibers" theory, Shepherd, 1994), states that taste is coded by the pattern of activity across a large population of receptors. The second one is called the labeled line theory and states that a given receptor is tuned to one specific taste.

At a perceptual level, the continuum of possible tastes is limited by four or five primary tastes: salty, bitter, sweet, sour, and the recently discovered umami (from the Japanese "delicious"), which corresponds, roughly, to the taste of glutamate.

\subsection{Trigeminal system}

The third neural component of flavor is the trigeminal system (see, Kandel et al., 2000). It involves the fifth cranial nerve also called the trigeminal nerve. As its name indicates (trigeminal means "triplets" in Latin), the trigeminal innervates most of the face and comprises three main branches: ophthalmic, mandibular, and maxillary which are all relevant to flavor. How the trigeminal system codes information is still obscure. It uses free endings located inside the mouth and the nose. But, it is possible that at least some of these free endings express receptors akin in structure to the smell receptors.

The trigeminal nerve makes synapses at the level of the spinal trigeminal nucleus and at the level of the principal sensory nucleus located respectively in the spine and the pons. These neurons, in turn, make synapses at the level of the thalamus (as almost all sensory information does) and then finally project in the face area of the primary somatic sensory cortex. The trigeminal system conveys information linked to several sensory modalities: touch, proprioception, temperature, and nociception. These include the texture and consistence of food as well as chemical irritation. In fact, the trigeminal system is stimulated by several categories of chemical substances including any pungent substance such as alcohol, ammonia, menthol, and capsaicin (from chili pepper), and also high concentrations of almost any odorant mixture. One of the functions of the trigeminal system seems to protect the organism from the effect of harmful substances as indicated by the effect of its stimulation: pain, sweating, tears, and running nose. And, also, the stimulation of the trigeminal system seems to induce inhibition of the smell system (possibly to protect it from the effects of over stimulation).

Even though the trigeminal system functions as a detector of harmful substances, human beings seem quite willing to indulge in its (excessive) stimulation as illustrated by our "inordinate proclivity" for chili pepper (or other capsaicin products), mustards, as well as carbonated beverages. Carsten and collaborators (in press) suggest several possible (nonexclusive) explanations for this paradoxical preference. They encompass a wide range, and go from the psychological (e.g., cognitive reinforcement, social pressure, "thrill-seeking", or even plain masochism) to the physiological (e.g., endorphin release, addiction).

\subsection{Flavor as synesthesia}

Thus flavor seems to involve three systems which differ by their anatomy, by the number of elementary dimensions they can code (from four or five tastes to 100,000 smells), and even by their function. It is therefore tempting to infer that these systems are also functionally independent. So, for example, a subject describing an odor as being sweet would be expressing, in fact, simply that this odor is the odor of something sweet but not that the odor itself is sweet. In other words, sweetness comes from taste and not from smell, and the apparent integration reflects, at best, a learned association.

However, anatomically independent systems can be cognitively or psychologically integrated. This point is clearer if we take the example of vision which is the most studied and best known perceptual system. It comprises two main subsystems themselves made of several independent modules (see, e.g., O’Toole, Roark, $\&$ Abdi, in press). In fact, these two main subsystems are already separated at the level of the retina. The first one is the magnocellular system, or "where" system. It processes information related to motion, depth, contrast, and position. It corresponds to a dorsal cortical pathway involving essentially the parietal lobes.

The second subsystem is the parvocellular system, also called the "what" system. It processes information related to shape and color. It corresponds to a ventral cortical pathway involving essentially the temporal lobes. Movement and localization are processed by two different modules of the where-system. Colors, object recognition, and face recognition correspond to different modules of the what-system.

The independence of these modules and systems can be assessed by clinical (i.e. neuropsychological) dissociations. Here, one system becomes non-functional whereas the other systems are intact. For example, some patients cannot perceive moving objects anymore but can perceive static ones. Inversely, other patients can perceive moving objects but cannot perceive static ones. Other specific dissociations include agnosia (loss of 
object recognition), prosopagnosia (loss of face recognition), or cerebral achromatopsia (inability to perceive colors). This last dissociation is beautifully illustrated by the case of Mr. I. described by Sacks (1995). Mr. I was an abstract painter who liked to use color. After a stroke destroyed essentially a small brain region in the occipital lobe called V4, Mr. I. lost all perception and memory for colors. His world became grey. This illustrates that V4 is the brain area responsible for the sensation of color. Visual systems can also be dissociated experimentally in animals by lesioning localized regions of the brain. For example, monkeys with temporal lesions are unable to perform an object discrimination task but can perform a landmark discrimination task. By contrast, monkeys with a dorsal lesion can perform an object discrimination task but cannot perform a landmark discrimination task. These functional dissociations are also supported by the recent use of brain imagery techniques both in humans and in animals (see Posamentier \& Abdi, in press, for a review).

So vision systems are clearly anatomically independent and easily dissociable. However, as our experience illustrates, we do not perceive the types of information coming from these systems has being independent. For example, we perceive the position, the shape, the color, and the movement of an object as indissociable properties of the object. In other words, vision cognitively integrates its independent neuroanatomical systems. Fodor (1983) uses the term encapsulated to refer to such integrated systems. Along the same lines, Pylyshyn (1999), in a recent review, describes vision as being an impenetrable system to imply that we do not have a conscious access to the neuroanatomy of vision, but have access only to its cognitive aspects.

But what about flavor? Recent work suggests that the subjective experience of flavor is created through the integration of taste, smell, and trigeminal stimulations. This integration seems to be performed by a specific brain region called the orbitofrontal cortex (see Fig. 1). Both brain imagery in humans (Royet et al., 1999) as well as single cells recordings in primates (Rolls, 1999) and other mammals (Schoenbaum, Chiba, \& Gallagher, 1999) point to the integrative role of this region. The orbitofrontal cortex receives projections from the olfactory cortex, the gustatory cortex, and the face primary sensory cortex (i.e., trigeminal projections, see Rolls, 1999, for a review). It also receives projections from the amygdala and the inferior temporal visual cortex. In fact, the orbitofrontal cortex is the first place in the brain where taste, smell, trigeminal information, and vision can all interact (see Fig. 2). In addition, Rolls and his collaborators (Rolls, Critchley, Mason, \& Wakeman, 1996; Rolls, Sienkiewicz, \& Yaxley, 1989) have shown that some cells in the orbitofrontal cortex not only integrate the three components of flavor, but also react to the current cognitive aspects of food intake such as appetence or reward value. The recent work of this group supports the notion that the orbitofrontal cortex is the brain center creating the psychological notion of flavor.

If the orbitofrontal is indeed creating flavor as the result of the integration of several systems, then we can expect to find behavioral instances of such an integration. In fact, cross modality interactions such as odor/ taste interactions have been well documented in the literature. For example, Frank and Byram (1988) showed that, for the same sugar concentrations, subjects perceive whipped cream with strawberry aroma as being sweeter than whipped cream alone. However, this type of interactions seems to involve two components (Nguyen, 2001). The first component would correspond to a response bias called halo-dumping by Clark and Lawless (1994). Halo dumping occurs when subjects are provided with only one intensity scale (sweetness) to rate a mixture of two sensations (sugar and strawberry). Forced to use one scale for two sensations, the subjects "dump" the second sensation onto the only available scale. This dumping effect disappears as soon as subjects are provided with a scale for each sensation. The second component, by contrast, would correspond to a real integration as suggested by Prescott (1999). Whereas the first component can intervene in rating any arbitrary combinations, the second one occurs only in familiar combinations. To evaluate this hypothesis, Nguyen, Valentin, Chrea, Ly, and Sauvageot (2001) compared the rating patterns obtained for one familiar combination (i.e., lemon-acid) and one unfamiliar combination (i.e., vanilla-acid). They found that for the familiar combination, subjects showed interaction even when provided with the two appropriate scales. However, providing subjects with a second scale eliminates the apparent interaction for the unfamiliar combination. And therefore, Nguyen et al concluded that real integration can occur, but that this is not always the rule.

But should we go as far as calling the orbitofrontal cortex the brain "cognitive food center?" In fact, this is probably not that simple, as the beautiful case of Phineas Gage illustrates (Harlow, 1848, 1868; Damasio, 1994). Phineas Gage, a railroad worker, who was 28 years old in 1848 , was considered a "most efficcient and capable man." He was working as a railroad technician, and was in charge of the difficcult task of blasting a way through the mountains (using dynamite). In the process, an accident occurred when an explosion started unexpectedly and propelled an iron rod through his skull. This destroyed his left orbitofrontal cortex. Harlow, a town physician who examined Gage immediately after the accident found him conscious and even able to describe the accident. He even found Gage perfectly normal and rational. Within 2 months Gage was pronounced cured because his movements, coordination, vision, hearing, and language were normal. But his personality had 
changed dramatically. To quote Harlow, "the equilibrium between his intellect and his animal propensities has been destroyed." Gage was not Gage anymore: He drifted, drank, and indulged in socially inappropriate behavior (Harlow indicates that "Ladies were not advised to stay in his company..."). More recently, in his popular book Descartes' Error, Damasio (1994) describes several similar cases. In fact, the orbitofrontal cortex seems to be involved in the integration of emotional and cognitive functions needed for complex decision making (see Schoenbaum et al., 1999), and flavor is just one part of the story.

\section{Part II: what can cognitive psychology learn from sensory evaluation?}

Sensory evaluation remains essentially an applied field, and therefore, it cannot afford to lose contact with the real world. As such, it stresses the importance of keeping stimuli naturalistic and taking into account individual differences. It can also open new fields of investigation or even open fresh theoretical perspectives.

\subsection{Keeping the world real: a rose is a rose is a rose}

In sensory evaluation, we need to use the real product because too much control might give clear answers to questions about which we do not really care. This is a truism for sensory evaluation professionals, but not always for cognitive psychologists. But, having worked on face processing for the last 20 years or so, I find this problem very familiar. In fact, experimental psychologists had to deal with a similar one with the faces used as experimental stimuli: In the 80 's, because of technical limitations, it was difficult-if not impossible-to manipulate and control real faces (remember, computers were a luxury in those days, and the word, or even the concept, of "morphing" did not even exist). It was then standard practice to use artificial faces made of arbitrary features as illustrated in Fig. 3.

As the faces were made of features, subjects were processing them as a collection of features rather than as a configuration (see Bartlett, Searcy, \& Abdi, in press, for a recent review). With faces being a bunch of features, then the orientation of the faces should have
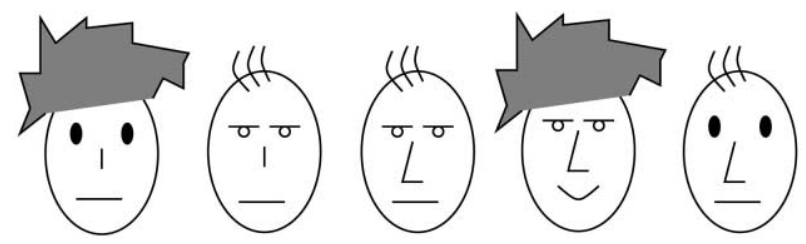

Fig. 3. A set of artificial faces made of some arbitrary features (from Abdi, Valentin, \& Edelman, 1999). no influence on the way they are analyzed. For example, the subjective similarity of two faces should be the same upside-down or right side-up as illustrated in Fig. 4. It is easy to see in Fig. 5 that this does not work for real faces (Thompson, 1980).

\subsection{Keeping the people real: experts and novices revisited}

Cognitive psychologists have studied the problem of intellectual expertise quite extensively, but have neglected the problem of perceptual expertise. On the other hand, sensory evaluation is obviously concerned with perceptual expertise. Therefore, comparing results coming from both field, can extend the understanding of the benefits of expertise.
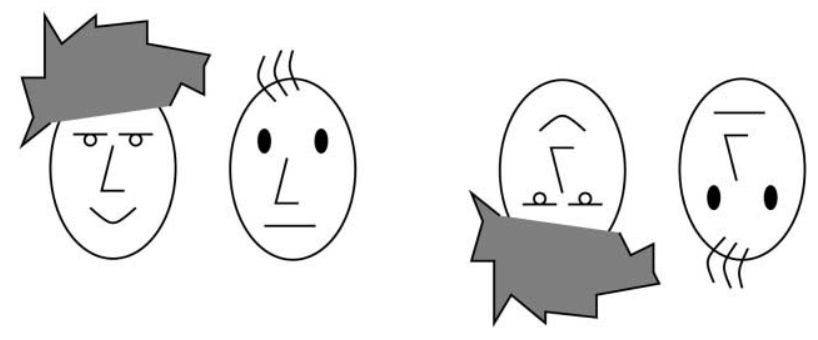

Fig. 4. The similarity of two artificial faces does not change much when they are presented right side-up or upside-down (faces from Abdi et al., 1999).
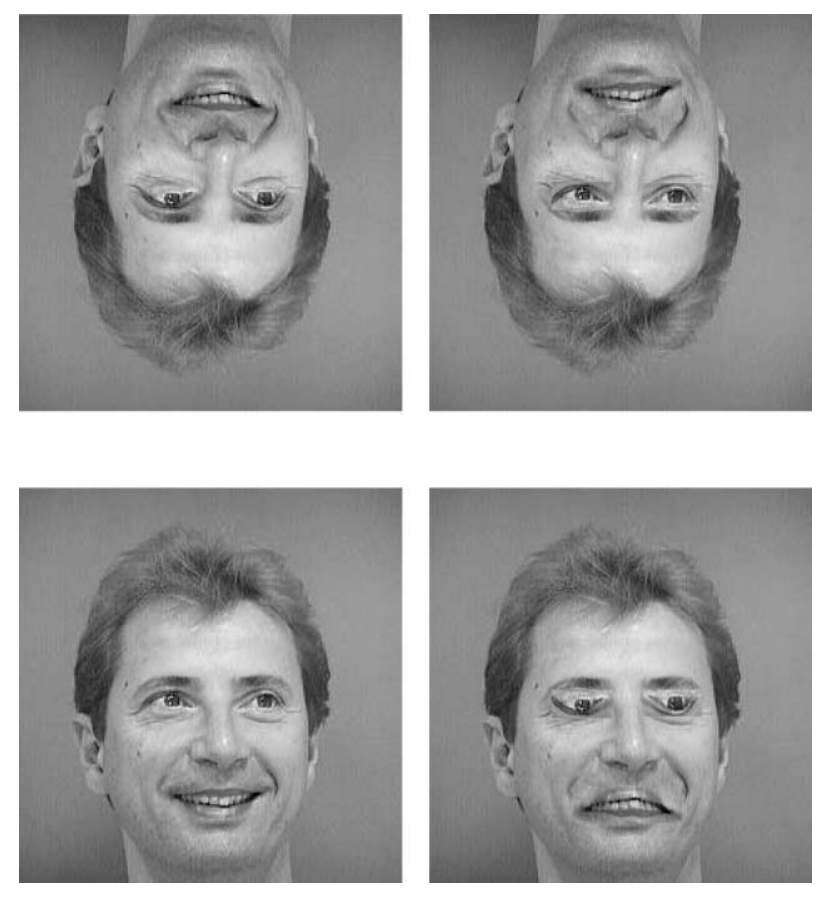

Fig. 5. The similarity of two real faces does change a lot when they are presented right side-up or upside-down (from Valentin, Edelman, \& Abdi, 1998). 


\subsection{Intellectual experts: chess players and mathematicians}

Intellectual experts differ dramatically from novices. Take the example of expert chess players such as grand masters. Not only do they play incredibly better than novices, but their expertise extends to other connected domains. For instance, when shown, for a short time, a chess board showing an intermediate state of a game, experts are able, on the average, to correctly place back from memory 26 pieces on the board (Chase \& Simon, 1973). By contrast, novices can barely place four pieces correctly. This difference of performance is due to expertise itself and not just to experts having a bigger memory: Put the pieces randomly on the chess board, and the experts perform no better than the novices. Grand masters, who have learned thousands of games, have memorized implicitly a very large number of possible configurations of pieces (The "positions" in chess jargon, or "chunks" in psychology jargon). So in a memory task, experts performed better because they memorized the configurations rather than the pieces.

Mathematicians can be very impressive also. For example, Aitkin, a British mathematician (see Hunter, 1977), could perform mental computations at spectacular speed (for human beings at least). When asked to compute mentally the square root of 851 , Aitkin gave the correct values (for their number of decimals) of 29.17 after $2 \mathrm{~s}$ and 29.17190429 after $10 \mathrm{~s}$. Ramanujan, who is often called the Mozart of mathematics, was even more impressive (Kanigel, 1991). When told by Hardy - a famous British mathematician himself (see Hardy, 1967) - that 1729 was a boring number, he immediately answered: "No, it's not, because 1729 is the smallest integer being the sum of two cubes in two different ways" $\left(1729=10^{3}+9^{3}=12^{3}+1^{3}\right)$.

So intellectual experts are much better than novices when tested in their domain of expertise. But, what about perceptual tasks?

\subsection{Perceptual experts: wine and beer}

Look at wine experts: how much do they differ from novices on perceptual tasks? To address this question, Valentin, Pichen, de Boiskerbert, and Abdi (2000) compared experts and novices on a discrimination task involving 14 French red wines and one Californian red wine. Subjects had to smell pairs of glasses of wine and decide if the same wine was in the glasses or not. Valentin et al. found that experts were only slightly better than novices; and both experts as well as novices performed better than chance. Curiously, subjects performed identically whether the different wines were from the same region or from different regions.

Beer experts look a bit better at a first glance. For example, Chollet (2000) compared novices and experts on a simple discrimination task. Her subjects had to taste samples of beers with aromas added (e.g., banana, honey, metallic) and decide if pairs of samples were identical or not. Experts were trained to detect and identify a set of aromas when tasting beers for 2 years previous to the experiment. When experts were tested with the aromas they had previously learned, they outperformed novices. But this advantage of expertise disappeared with new aromas.

On the whole, wine and beer experts do perform better than novices on perceptual tasks, but not that much better. However, experts clearly outperform novices as soon as language is involved: When asked to describe wine or beer, experts use a richer and more precise vocabulary than novices (Chollet \& Valentin, 2000, in press; Gawell, 1997; Lawless, 1984; Solomon, 1990). Like intellectual experts, wine and beer experts organize their knowledge and can rely on this organization for creating efficcient retrieval cues. For example, wine experts organize their knowledge of wine sensory properties around grape varieties. And like chess experts, their memory is better when the description of a wine matches real varieties than when attributes are randomly grouped (Hugson \& Boakes, 2001a, 2001b).

Comparing intellectual experts with sensory experts suggests that expertise exits in different types. Sensory experts are impressive indeed, but much less than intellectual experts. Finding the source of this difference is likely to be the source of much work to come. It is, however, tempting to speculate that this difference reflects a deeper difference between perception and cognition. Our perceptual systems are, from an evolutionary perspective, quite old and therefore are likely to have been optimized by evolution. And so expertise cannot improve much on such well optimized systems. By contrast, language and higher cognitive processes seem specific to human beings (even if precursors can be found in other animals) and hence are relatively recent, and here expertise has more room for improving performance.

\section{Conclusion}

As the examples described here show, cognitive psychology and sensory evaluation can benefit a lot from each other and, in fact, already do. I have considered only a small subset of their possible domains of interactions. It is likely that the problem of how different sensorial modalities (smell, taste, touch, vision) are integrated to form a cognitively relevant qualia will be one important domain of common interest.

\section{Acknowledgements}

Thanks are due to Dominique Valentin, Claire Sulmont-Rosssé, Sylvie Issanchou, André Holley, Lori 
Stephens, Jay Dowling, Mette Posamentier, Earl Carsten, and two anonymous reviewers for their help in preparing this paper.

\section{References}

Abdi, H., Valentin, D., \& Edelman, B. (1999). Neural networks. Thousand Oaks, CA: Sage.

Bartlett, J. A., Searcy, J., \& Abdi, H. (in press). What are the routes to face recognition? In M. Peterson, \& G. Rhodes (Eds.), Analytic and holistic processes in the perception of faces, object, and scenes. Norwood, NJ: Ablex.

Carsten, E., Carsten, M.I., Dessirier, J.M., O'Mahony, M., Simons, T., Sudo, M., \& Sudo, S. (in press). It hurts so good: oral irritation by spices and carbonated drinks and the underlying neural mechanisms. Food Quality and Preference.

Chase, W. G., \& Simon, H. A. (1973). The mind's eye in chess. In W. G. Chase (Ed.), Visual information processing. New York: Academic Press.

Chollet, S. (2000). Entraînement individuel versus entraînement en groupe, experts versus novices: quelles sont les différences? Application au domaine de la biére. Doctoral dissertation, Universite de Dijon, France, 2000.

Chollet, S., \& Valentin, D. (2000). Le degré d'expertise a-t-il une influence sur la perception olfactive: quelques éléments de réponse dans le domaine du vin. L'Année Psychologique, 100, 11-36.

Chollet, S., \& Valentin, D. (2001). Impact of training on beer flavour perception and description: Are trained and untrained subjects really different? Journal of Sensory Studies, 16, 601-618.

Clark, C. C., \& Lawless, H. T. (1994). Limiting response alternatives in Cognitive psychology and sensory evaluation 20 time-intensity scaling: an examination of the Halo-dumping effect. Chemical Senses, 19, 583-594.

Damasio, A. R. (1994). Descartes' error: emotion, reason and the human brain. New York: Avon Books.

Doty, R. L. (2001). Olfaction. Annual Review of Psychology, 52, 423-452.

Fodor, J. A. (1983). The modularity of mind. Cambridge, MA: MIT Press.

Frank, R. A., \& Byram, J. (1988). Taste-smell interactions are tastant and odorant dependent. Chemical Senses, 13, 445.

Gawell, R. (1997). The use of language by trained and untrained experienced wine tasters. Journal of Sensory studies, 12, 267-286.

Hardy, G. H. (1967). A mathematician's apology. Cambridge: Cambridge University Press.

Harlow, J. M. (1848). Passage of an iron rod through the head. Boston Medical and Surgical Journal, 39, 389.

Harlow, J. M. (1868). Recovery from the passage of an iron bar through the head. Publications of the Massachusetts Medical Society, 2, 327-347.

Holley, A. (1999). Recent advances in mechanisms of odor perception. Water Science \& Technology, 40, 79-84.

Hunter, I. M. L. (1977). Mental calculations. In P. N. Johnson-Laird, \& P. C. Wason (Eds.), Thinking: readings in cognitive science. Cambridge, MA: Cambridge University Press.

Hugson, A., \& Boakes, R. (2001a). Perceptual and cognitive elements of wine tasting expertise. Australian Journal of Psychology, 53, 103-108.

Hugson, A., \& Boakes, R. (2001b). The knowing nose: the role of knowledge in wine expertise. Paper presented at the 4th Pangborn Sensory Science Symposium, Dijon, France.

Kandel, E. R., Schwartz, J. H., \& Jessell, T. M. (2000). Principles of neural science (4th ed.). New York: McGraw-Hill.

Kanigel, R. (1991). The man who knew infinity: a life of the genius Ramanujan. Washington, DC: Washington Square Press.

Kauer, J. S., \& White, J. (2001). Imaging and coding in the olfactory system. Annual Review of Neurosciences, 24, 963-979.
Koster, E. P. (1983). Uses and misuses of sensory analysis: a psychologist point of view. Reference, 129-137.

Laurent, G., Stopfer, M., Friedrich, R. W., Rabinovich, M. I., Volkovskii, A., \& Abarbanel, H. D. I. (2001). Odor encoding as an active, dynamical process: experiments, computation, and theory. Annual Review of Neurosciences, 24, 263-297.

Lawless, H. T. (1984). Flavor description of white wines by "experts" and non-experts wine consumers. Journal of Food Sciences, 49, 120 123.

Lawless, H. T., \& Heymann, H. (1999). Sensory evaluation of food: principles and practices. New York: Chapmann and Hall.

Mombaerts, P. (2001). How smell develops. Nature Neuroscience, 4, 1192-1198.

Nguyen, D. (2001). Contribution à l'étude des interactions entre entrées sensorielles: l'effet de la présence d'une odeur sur la perception d'une saveur. Doctoral dissertation, Université de Dijon, France, 2001.

Nguyen, D., Valentin, D. Chrea, C., Ly, M.H., \& Sauvageot, F. (2001). When does smell enhance taste? Effect of culture and odorant tastant relationship. Poster presented at the 4th Pangbotn Sensory Science Symposium, Dijon, France.

O'Toole, A.J., Roark, D.A., \& Abdi, H. (in press). Recognizing moving faces: a psychological and neural synthesis. Trends in Cognitive Science, 6, 261-266.

Posamentier, M.T., \& Abdi, H. (in press). Processing facial information: evidence from behavioral, experimental, and brain imaging studies. Neuropsychology Review.

Prescott, J. (1999). Flavour as a psychological construct: implications for perceiving and measuring the sensory qualities of foods. Food Quality and Preference, 10, 349-356.

Pylyshyn, Z. (1999). Is vision continuous with cognition? The case for cognitive impenetrability of visual perception? Behavioral and Brain Sciences, 22, 341-423.

Rolls, T. E. (1999). The brain and emotion. Oxford: Oxford University Press.

Rolls, T. E., Critchley, H., Mason, R., \& Wakeman, E. A. (1996). Orbitofrontal cortex neurons: role in olfactory and visual association learning. Journal of Neurophysiology, 75, 1970-1981.

Rolls, T. E., Sienkiewicz, Z. J., \& Yaxley, S. (1989). Hunger modulates the responses to gustatory stimuli of single neurons in the caudalateral orbitofrontal cortex of the macaque monkey. European Journal of Neurosciences, 1, 53-60.

Royet, J. P., Koenig, O., Gregoire, M. C., Cinotti, L., Lavenne, F., Le Bars, D., Costes, N., Vigouroux, M., Farget, V., Sicard, G., Holley, A., Mauguiere, F., Comar, D., \& Froment, J. C. (1999). Functional anatomy of perceptual ans semantic processing for odors. Journal of Cognitive Neurosciences, 11, 94-109.

Sacks, D. (1995). An anthropologist from Mars: Seven paradoxical tales. New York: Vintage Books.

Shepherd, G. M. (1994). Neurobiology. Oxford: Oxford University Press.

Schoenbaum, G., Chiba, A. A., \& Gallagher, M. (1999). Neural encoding in orbitofrontal cortex and basolateral amygdala during olfactory discrimination learning. The Journal of Neurosciences, 19, 1876-1884.

Solomon, G. F. A. (1990). Psychology of novice and expert wine talk. American Journal of Psychology, 103, 495-512.

Thompson, P. (1980). Margaret Thatcher: A new illusion? Perception, 9, 483-484.

Valentin, D., Edelman, B., \& Abdi, H. (1998). Computational and Neural network models of face processing. Journal of Biological System, 6, 213-219.

Valentin, D., Pichen, M., de Boisherbert, V., \& Abdi, H. (2000). What's in a wine name: when and why do wine experts perform better than novices. Poster presented at the 41st Psychonomic Meeting, New Orleans, USA. 